\title{
Weighted Shifts on Directed Semi-Trees: an Application to Creation Operators on Segal-Bargmann Spaces
}

\author{
Witold Majdak $^{1} \cdot$ Jerzy Bartłomiej Stochel ${ }^{1}$
}

Received: 13 November 2014 / Accepted: 21 April 2015 / Published online: 16 May 2015

C The Author(s) 2015. This article is published with open access at Springerlink.com

\begin{abstract}
We extend the notion of a weighted shift on a directed tree to the case of a more general graph which we call a directed semi-tree. Some basic properties of such operators are investigated. It is shown that a generalized creation operator on the Segal-Bargman space is unitarily isomorphic to a weighted shift on a directed semi-tree of a particular form.
\end{abstract}

Keywords Directed tree · Weighted shift · Segal-Bargmann space ·

Creation operator

Mathematics Subject Classification Primary 47A35 - 47B65; Secondary 47B20

\section{Introduction}

Relating Hilbert space operators to directed graphs can provide some efficient new tools in operator theory. The study of non-selfadjoint operators can be, for example, successfully realized through connecting directed graphs with corresponding adjacency bounded operators. Such an approach appeared in [8] and was subsequently developed in [7]. In order to obtain significantly more information about operators

\section{Communicated by Yuri Kondratiev.}

$凶$ Witold Majdak

majdak@agh.edu.pl

Jerzy Bartłomiej Stochel

stochel@agh.edu.pl

1 Faculty of Applied Mathematics, AGH University of Science and Technology, Al. A. Mickiewicza 30, 30-059 Krakow, Poland 
related to graphs Jabłoński, Stochel, and Jung focused attention in [9] on directed trees. They introduced the notion of a weighted shift on a directed tree and effectively investigated properties of a class of such (unbounded) operators. We refer to [4-6,10-12] for further results on the subject. It should be noticed that this concept substantially generalized the notion of a classical weighted shift (see, for example, [15] or [13]). In the present paper we extend the definition of a weighted shift on a directed tree from [9] to the case of a more general graph which we propose to call a directed semi-tree. The idea of broadening this definition to a larger class of operators was motivated by the fact that the properties of generalized creation operators on Segal-Bargman spaces cannot be described by using the theory developed in [9]. Here we obtain counterparts of some results included therein in this new setting. It is worth noting that most of arguments of [9, Chapter 3] do not transfer automatically to the case of directed semi-trees. Therefore, in the paper we have to apply some alternative proof techniques. It turns out that our results embrace generalized creation operators.

The paper is organized as follows. In Sect. 2 we set up notation and terminology of the graph theory which is used throughout this work. We recall some properties of directed trees and next introduce a concept of a directed semi-tree. In Sect. 3 we define $n$-finite and infinite Bargmann graphs, discuss their properties, and show that they are in fact directed semi-trees. In Sect. 4 we show that Segal-Bargmann spaces can be regarded as $l^{2}$-Hilbert spaces defined on vertices of the Bargmann graphs. The main results of the paper are to be found in Sects. 5-7. In Sect. 5 we generalize the notion of a weighted shift on a directed tree from [9] to the case of a directed semi-tree, and next we prove that such weighted shifts have, in some situations, similar properties to those on trees. Section 6 is devoted to showing that the generalized creation operators, defined by Bargmann in [2] and [3], are unitarily isomorphic to weighted shifts on the respective Bargmann graphs. Finally, in Sect. 7 we give some sufficient conditions for the orthonormal basis of the $l^{2}$-type space to be a core for a weighted shift on a directed semi-tree. We also observe that these conditions are fulfilled by the generalized creation operators. The differences between weighted shifts defined on directed trees and those defined on directed semi-trees are highlighted in Sects. 5 and 7. The inspiration for writing this paper comes from [9] and [17].

\section{Directed Trees and Directed Semi-Trees}

Throughout the paper, we employ the standard terminology of the graph theory which was also utilized in [9].

We say that a pair $\mathcal{G}=(V, E)$ is a directed graph if $V$ is a nonempty set and $E$ is a subset of $(V \times V) \backslash\{(v, v): v \in V\}$. Put

$$
\widetilde{E}=\{\{u, v\} \subset V:(u, v) \in E \text { or }(v, u) \in E\} .
$$

We say that a member of $V$ is a vertex of $\mathcal{G}$, a member of $E$ is an edge of $\mathcal{G}$, and finally a member of $\widetilde{E}$ is an undirected edge of $\mathcal{G}$. If $W$ is a nonempty subset of $V$, then the pair $(W, E \cap(W \times W))$ is also a directed graph which is called a directed subgraph of $\mathcal{G}$. A directed graph $\mathcal{G}$ is said to be connected if for every two distinct 
vertices $u$ and $v$ of $\mathcal{G}$ there exists a finite sequence $v_{1}, \ldots, v_{n}(n \geq 2)$ of vertices of $\mathcal{G}$ such that $u=v_{1},\left\{v_{j}, v_{j+1}\right\} \in \widetilde{E}$ for all $j=1, \ldots, n-1$, and $v_{n}=v$. Such a sequence is called an undirected path joining $u$ and $v$. Let

$$
\begin{array}{ll}
\operatorname{Chi}(u):=\{v \in V:(u, v) \in E\} & \text { for } u \in V, \\
\operatorname{Par}(v):=\{u \in V:(u, v) \in E\} & \text { for } v \in V .
\end{array}
$$

We say that a member of $\operatorname{Chi}(u)$ is a child of $u$ and a member of $\operatorname{Par}(v)$ is a parent of $v$. If for a vertex $v \in V$ there exists a unique vertex $u \in V$ being its parent (i.e. $\operatorname{Par}(v)=\{u\})$, then we briefly write $u=\operatorname{par}(v)$. A finite sequence $v_{1}, \ldots, v_{n}$ of distinct vertices of $\mathcal{G}$ is said to be a circuit of $\mathcal{G}$ if $n \geq 2,\left(v_{j}, v_{j+1}\right) \in E$ for all $j=1, \ldots, n-1$, and $\left(v_{n}, v_{1}\right) \in E$. A vertex $v$ of $\mathcal{G}$ is called a root of $\mathcal{G}$ if there is no vertex $u$ of $\mathcal{G}$ such that $(u, v)$ is an edge of $\mathcal{G}$. We denote by $\operatorname{Root}(\mathcal{G})$ the set of all roots of $\mathcal{G}$. If $\operatorname{Root}(\mathcal{G})$ is a one-element set, then its unique element is denoted by root. For $W \subset V$, we set $W^{\circ}=W \backslash \operatorname{Root}(\mathcal{G})$ and write card $(W)$ for the cardinality of $W$.

Let us recall Proposition 2.1.1 of [9].

Proposition 2.1 Let $\mathcal{G}$ be a directed graph satisfying the following conditions:

(i) $\mathcal{G}$ is connected,

(ii) for each vertex $v \in V^{\circ}$, the set $\operatorname{Par}(v)$ has only one element.

Then the set $\operatorname{Root}(\mathcal{G})$ contains at most one element.

Let $\mathcal{G}$ be a directed graph. Given a set $W \subset V$, we put

$$
\operatorname{Chi}(W)=\bigcup_{w \in W} \operatorname{Chi}(w), \quad \operatorname{Par}(W)=\bigcup_{w \in W} \operatorname{Par}(w)
$$

Define also

$$
\mathrm{Chi}^{\langle 0\rangle}(W)=W, \quad \operatorname{Chi}^{\langle n+1\rangle}(W)=\operatorname{Chi}\left(\mathrm{Chi}^{i n\rangle}(W)\right) \text { for } n=0,1, \ldots,
$$

and

$$
\operatorname{Des}(W)=\bigcup_{n=0}^{\infty} \operatorname{Chi}^{\langle n\rangle}(W)
$$

The members of $\operatorname{Des}(W)$ are called descendants of $W$. If $W=\{v\}$, then, for brevity, we write $\operatorname{Des}(v)$ instead of $\operatorname{Des}(\{v\})$. Note that if $\mathcal{G}$ is a connected graph and $\operatorname{Root}(\mathcal{G})=$ \{root\}, then

$$
\operatorname{Des}(\text { root })=V \text { and } \operatorname{Par}(\text { root })=\emptyset \text {. }
$$

Definition 2.2 We say that a directed graph $\mathcal{G}=(V, E)$ is a directed tree if it has no circuits and satisfies the conditions (i) and (ii) of Proposition 2.1. 
Let us recall some properties of directed trees given in [9] (see Propositions 2.1.2 and 2.1.4 therein, respectively) that we will utilize in our further considerations.

Proposition 2.3 If $\mathcal{G}$ is a directed tree, then $\operatorname{Chi}(u) \cap \operatorname{Chi}(v)=\emptyset$ for all distinct $u, v \in V$ and $V^{\circ}=\bigcup_{v \in V} \operatorname{Chi}(v)$.

Proposition 2.4 If $\mathcal{G}$ is a directed tree, then for all distinct $u, v \in V$ there exists $w \in V$ such that $u, v \in \operatorname{Des}(w)$.

Remark 2.5 The reader may easily convince himself that if $\mathcal{G}$ is a directed graph, then the following conditions are equivalent:

(i) $\operatorname{Chi}(u) \cap \operatorname{Chi}(v)=\emptyset$ for all distinct $u, v \in V$,

(ii) $\operatorname{card}(\operatorname{Par}(v))=1$ for each $v \in V^{\circ}$.

Following these preliminary results, we can introduce a more general notion than a directed tree. It plays a pivotal role in our paper.

Definition 2.6 We say that a directed graph $\mathcal{F}=(V, E)$ is a directed semi-tree if

(i) it has no circuits,

(ii) $\mathcal{F}$ is connected,

(iii) $\operatorname{card}(\operatorname{Chi}(u) \cap \operatorname{Chi}(v)) \leq 1$ for all distinct $u, v \in V$,

(iv) for all $u, v \in V$, there exists $w \in V$ such that $u, v \in \operatorname{Des}(w)$.

Note that directed trees fall within the scope of Definition 2.6, because for such graphs conditions (iii) and (iv) are satisfied by Proposition 2.3 and 2.4, respectively. In view of Remark 2.5 a relation between directed trees and directed semi-trees can be characterized as follows.

Proposition 2.7 If $\mathcal{F}=(V, E)$ is a directed semi-tree, then $\mathcal{F}$ is a directed tree if and only if $\operatorname{Chi}(u) \cap \operatorname{Chi}(v)=\emptyset$ for all distinct $u, v \in V$.

For completeness of exposition, let us discuss relations between conditions appearing in Definition 2.6.

Remark 2.8 Suppose that the conditions (i) and (ii) of Definition 2.6 are satisfied. If there exists $v \in V^{\circ}$ which has more then one parent, then, in general, none of conditions (iii) and (iv) holds. To see this, one may consider fairly easy examples of graphs such as (a) and (b), respectively, in Fig. 1. We also point out that neither (iii) implies (iv) nor (iii) follows from (iv). Indeed, it is enough to consider graphs (b) and (c), respectively, illustrated in Fig. 1.

As we see below, a directed semi-tree, similarly as a directed tree, cannot have more than one root.

Proposition 2.9 If $\mathcal{F}=(V, E)$ is a directed graph which is connected and satisfies the condition (iv) of Definition 2.6, then the set $\operatorname{Root}(\mathcal{G})$ contains at most one element. 
(a)

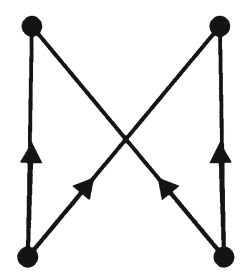

(b)

(c)

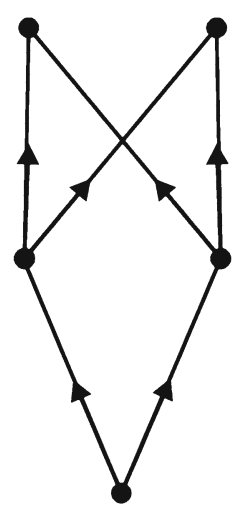

Fig. 1 Graphs illustrating Remark 2.8

Proof Suppose that, contrary to our claim, there exist distinct $u, v \in \operatorname{Root}(\mathcal{F})$. By (iv), we can find $w \in V$ such that $u, v \in \operatorname{Des}(w)$. Since $\mathrm{Chi}^{\langle 0\rangle}(\{w\})=\{w\}$, it follows that at least one of these roots, say $u$, belongs to $\mathrm{Chi}^{\langle n\rangle}(\{w\})$ for some $n \geq 1$. This means that $u$ has a parent, which is a contradiction.

We conclude this section with one more definition. A subgraph of a directed tree (resp. a directed semi-tree) $\mathcal{F}$ which itself is a directed tree (resp. directed semi-tree) is called a subtree (resp. a subsemi-tree) of $\mathcal{F}$.

The proofs of the following propositions are left for the reader.

Proposition 2.10 Let $\mathcal{G}=(V, E)$ be a directed tree. A subgraph $\mathcal{G}_{1}$ of $\mathcal{G}$ is a directed tree if and only if $\mathcal{G}_{1}$ is a connected graph.

Proposition 2.11 Let $\mathcal{F}=(V, E)$ be a directed semi-tree. A subgraph $\mathcal{F}_{1}=\left(V_{1}, E_{1}\right)$ of $\mathcal{F}$ is a directed semi-tree if and only if $\mathcal{F}_{1}$ is a connected graph and for all $u, v \in V_{1}$, there exists $w \in V_{1}$ such that $u, v \in \operatorname{Des}(w)$.

\section{The Bargmann Directed Semi-Trees}

Here and in all that follows, we shall use the following notation. By $\mathbb{N}, \mathbb{Z}_{+}$and $\mathbb{C}$ we mean the sets of all positive integers, non-negative integers, and complex numbers, respectively. The cardinality of $\mathbb{N}$ is denoted by $\aleph_{0}$.

Let

$$
V_{n}=\left\{\left(\alpha_{1}, \ldots, \alpha_{n}\right): \alpha_{i} \in \mathbb{Z}_{+}, i=1, \ldots, n\right\}, \quad n \in \mathbb{N}
$$

and

$$
\begin{aligned}
V_{\infty}= & \left\{\left(\alpha_{1}, \alpha_{2}, \ldots\right): \alpha_{i} \in \mathbb{Z}_{+}, i \in \mathbb{N}\right. \\
& \text { and card } \left.\left\{i \in \mathbb{N}: \alpha_{i} \neq 0\right\}<\aleph_{0}\right\}
\end{aligned}
$$


We equip these sets with natural algebraic operations:

$$
\begin{aligned}
& \alpha \pm \beta:=\left(\alpha_{1} \pm \beta_{1}, \ldots, \alpha_{n} \pm \beta_{n}\right), \quad \alpha=\left(\alpha_{1}, \ldots, \alpha_{n}\right), \beta=\left(\beta_{1}, \ldots, \beta_{n}\right) \in V_{n}, \\
& \alpha \pm \beta:=\left(\alpha_{1} \pm \beta_{1}, \alpha_{2} \pm \beta_{2}, \ldots\right), \quad \alpha=\left(\alpha_{1}, \alpha_{2}, \ldots\right), \beta=\left(\beta_{1}, \beta_{2}, \ldots\right) \in V_{\infty} .
\end{aligned}
$$

Next, we set

$$
\begin{aligned}
E_{n}= & \left\{(\alpha, \beta): \alpha, \beta \in V_{n} \text { and there exists } i \in\{1, \ldots, n\}\right. \\
& \text { such that } \left.\beta-\alpha=\delta_{i}\right\}, \quad n \in \mathbb{N},
\end{aligned}
$$

and

$$
E_{\infty}=\left\{(\alpha, \beta): \alpha, \beta \in V_{\infty} \text { and there exists } i \in \mathbb{N} \text { such that } \beta-\alpha=\delta_{i}\right\}
$$

where $\delta_{i}$ denotes a sequence from $V_{n}$ (resp. $V_{\infty}$ ) whose the only non-zero entry is 1 at the $i$-th position.

It is plain that $\mathfrak{B}_{n}:=\left(V_{n}, E_{n}\right), n \in \mathbb{N}$, and $\mathfrak{B}_{\infty}:=\left(V_{\infty}, E_{\infty}\right)$ are directed graphs. We call them the $n$-finite Bargmann graph and the infinite Bargmann graph, respectively. Each $\mathfrak{B}_{n}$ can be regarded as a subgraph of $\mathfrak{B}_{\infty}$ with the help of the natural identification

$$
V_{n} \ni\left(\alpha_{1}, \ldots, \alpha_{n}\right) \mapsto\left(\alpha_{1}, \ldots, \alpha_{n}, 0,0, \ldots\right) \in V_{\infty}
$$

An exemplary graph $\mathfrak{B}_{n}$ (for $n=3$ ) is illustrated in Fig. 2.

In what follows, we confine our attention to $\mathfrak{B}_{\infty}$, because most of properties of all $\mathfrak{B}_{n}$ 's can be described in an analogous way. If some property is typical only for $\mathfrak{B}_{\infty}$, we will pay the reader's attention to this fact.

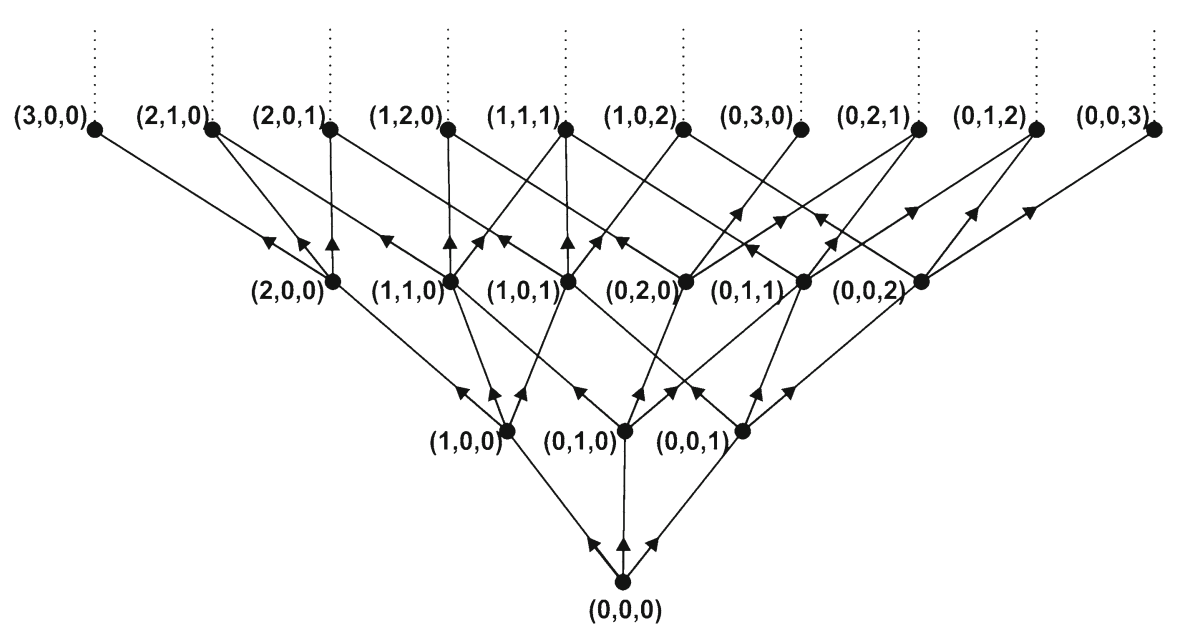

Fig. 2 A structure of the graph $\mathfrak{B}_{3}$ 
Denote by $\Theta$ the sequence from $V_{\infty}$ consisting of zeros only. For $\alpha, \beta \in V_{\infty}$, we write $\alpha \geq \beta$ if $\alpha_{i} \geq \beta_{i}$ for each $i \in \mathbb{N}$, and $\alpha>\beta$ if $\alpha \geq \beta$ and $\alpha \neq \beta$.

Let us discuss some properties of the Bargmann graph $\mathfrak{B}_{\infty}$.

Lemma 3.1 (i) Let $\alpha, \beta \in V_{\infty}$ and $\alpha>\beta$. Then there exits a finite sequence $\gamma_{1}, \ldots, \gamma_{m} \in V_{\infty}$ such that $\gamma_{1}=\beta,\left(\gamma_{i}, \gamma_{i+1}\right) \in E_{\infty}$ for $i=1, \ldots, m(m \geq 2)$ and $\gamma_{m}=\alpha$.

(ii) $\mathfrak{B}_{\infty}$ is a connected graph.

(iii) $\operatorname{Root}\left(\mathfrak{B}_{\infty}\right)=\{\Theta\}$.

(iv) $\operatorname{Des}(\Theta)=V_{\infty}$.

Proof (i) The set $\left\{i \in \mathbb{N}: \alpha_{i}>\beta_{i}\right\}$ is finite, so we can write it as $\left\{i_{1}, \ldots, i_{k}\right\}$ for some $k \in \mathbb{N}$. We define the required sequence $\gamma_{1}, \ldots, \gamma_{m}$, where $m=\sum_{i=1}^{\infty}\left(\alpha_{i}-\right.$ $\left.\beta_{i}\right)+1 \geq 2$, as follows:

$$
\begin{aligned}
& \gamma_{1}=\beta, \gamma_{2}=\beta+\delta_{i_{1}}, \ldots, \gamma_{\alpha_{i_{1}}-\beta_{i_{1}}+1}=\beta+\left(\alpha_{i_{1}}-\beta_{i_{1}}\right) \delta_{i_{1}} \\
& \gamma_{\alpha_{i_{1}}-\beta_{i_{1}}+2}=\beta+\left(\alpha_{i_{1}}-\beta_{i_{1}}\right) \delta_{i_{1}}+\delta_{i_{2}}, \ldots, \gamma_{m}=\alpha
\end{aligned}
$$

(ii) Take distinct $\alpha, \beta \in V_{\infty}$. If one of these elements equals $\Theta$, then the conclusion follows immediately from (i). Now suppose that $\alpha>\Theta$ and $\beta>\Theta$. By (i), there exist finite sequences $\gamma_{1}, \ldots, \gamma_{k}$ and $\tilde{\gamma}_{1}, \ldots, \tilde{\gamma}_{m}$ of vertices of $\mathfrak{B}_{\infty}$ such that $\gamma_{1}=\tilde{\gamma}_{1}=\Theta,\left(\gamma_{i}, \gamma_{i+1}\right) \in E_{\infty}$ for $i=1, \ldots, k-1,\left(\tilde{\gamma}_{j}, \tilde{\gamma}_{j+1}\right) \in E_{\infty}$ for $j=1, \ldots, m-1, \gamma_{k}=\alpha$ and $\tilde{\gamma}_{m}=\beta$. Thus $\left\{\tilde{\gamma}_{m}, \tilde{\gamma}_{m-1}\right\}, \ldots,\left\{\tilde{\gamma}_{2}, \tilde{\gamma}_{1}\right\},\left\{\gamma_{1}, \gamma_{2}\right\}$, $\ldots,\left\{\gamma_{k-1}, \gamma_{k}\right\}$ is an undirected path joining $\beta$ and $\alpha$, and so the graph $\mathfrak{B}_{\infty}$ is connected.

(iii) follows directly from (i), while (iv) is a consequence of (iii) and (2.1).

Lemma $3.2 \mathfrak{B}_{\infty}$ has no circuits.

Proof Suppose that, contrary to our claim, $\mathfrak{B}_{\infty}$ has a circuit. Take distinct $\alpha, \beta \in V_{\infty}$ belonging to this circuit, which can be described by the following directed path:

$$
\left(\alpha, \gamma_{1}\right), \ldots,\left(\gamma_{k}, \alpha\right)
$$

where $\gamma_{1}, \ldots, \gamma_{k} \in V_{\infty}(k \in \mathbb{N})$ and $\beta=\gamma_{l}$ for some $l \in\{1, \ldots, k\}$. Hence $\beta \geq \alpha$ and $\alpha \geq \beta$, and so $\alpha=\beta$, which is a contradiction.

The next result is obvious, so we omit its proof.

Lemma 3.3 For all $\alpha \in V_{\infty}$ and $\beta \in V_{\infty}^{\circ}$ the following assertions hold:

(i) $\operatorname{Chi}(\alpha)=\left\{\alpha+\delta_{i}: i \in \mathbb{N}\right\}$,

(ii) $\operatorname{card}(\operatorname{Chi}(\alpha))=\aleph_{0}$,

(iii) $\operatorname{Par}(\beta)=\left\{\beta-\delta_{i}: \beta_{i}>0, i \in \mathbb{N}\right\}$,

(iv) $\operatorname{Par}(\beta) \neq \emptyset$ and $\operatorname{card}(\operatorname{Par}(\beta))=\left\{i \in \mathbb{N}: \beta_{i} \neq 0\right\}<\aleph_{0}$. 
Remark 3.4 Note that in the case of $\mathfrak{B}_{n}(n \in \mathbb{N})$ assertion (ii) of Lemma 3.3 takes the form $\operatorname{card}(\operatorname{Chi}(\alpha))=n$.

Lemma 3.5 For all $\alpha, \beta \in V_{\infty}$ such that $\alpha \neq \beta$ the following assertions hold:

(i) $\operatorname{Chi}(\alpha) \cap \operatorname{Chi}(\beta) \neq \varnothing$ if and only if there exist distinct $i_{1}, i_{2} \in \mathbb{N}$ such that $\alpha+\delta_{i_{1}}=\beta+\delta_{i_{2}} \in \operatorname{Chi}(\alpha) \cap \operatorname{Chi}(\beta)$,

(ii) $\operatorname{card}(\operatorname{Chi}(\alpha) \cap \operatorname{Chi}(\beta)) \leq 1$.

Proof (i) follows from Lemma 3.3 (i).

(ii) If $\operatorname{Chi}(\alpha) \cap \operatorname{Chi}(\beta) \neq \emptyset$, then by (i), there exist distinct $i_{1}, i_{2} \in \mathbb{N}$ such that

$$
\alpha+\delta_{i_{1}}=\beta+\delta_{i_{2}} \in \operatorname{Chi}(\alpha) \cap \operatorname{Chi}(\beta),
$$

which yields $\alpha_{i}=\beta_{i}$ for each $i \in \mathbb{N} \backslash\left\{i_{1}, i_{2}\right\}$. Hence the pair $\left(i_{1}, i_{2}\right)$ is uniquely determined. This completes the proof.

Let us briefly conclude our discussion in the form of the following theorem.

Theorem 3.6 $\mathfrak{B}_{\infty}$ and $\mathfrak{B}_{n}(n \in \mathbb{N})$ are directed semi-trees.

Proof The assertion follows from a combination of Lemma 3.2, Lemma 3.1 (ii), Lemma 3.5 (ii), and Lemma 3.1 (iv).

In view of Proposition 2.11 and the above theorem we can regard each $\mathfrak{B}_{n}(n \in \mathbb{N})$ as a directed subsemi-tree of $\mathfrak{B}_{\infty}$.

\section{Segal-Bargmann Spaces as $l^{2}$-Hilbert Spaces on Directed Semi-Trees}

Let $\mathcal{F}=(V, E)$ be a directed semi-tree. We denote by $l^{2}(V)$ the Hilbert space of all complex square-summable functions on $V$ equipped with the standard inner product

$$
\langle f, g\rangle=\sum_{v \in V} f(v) \overline{g(v)}, \quad f, g \in l^{2}(V)
$$

For $u \in V$, we define $e_{u} \in l^{2}(V)$ to be the characteristic function of the oneelement set $\{u\}$ (i.e. $e_{u}(v)=1$ if $u=v$ and $e_{u}(v)=0$ if $u \neq v$ ). Then $\left\{e_{u}\right\}_{u \in V}$ is an orthonormal basis of $l^{2}(V)$. Note that $l^{2}(V)$ is a reproducing kernel Hilbert space [1], which is guaranteed by the reproducing property

$$
f(u)=\left\langle f, e_{u}\right\rangle, \quad f \in l^{2}(V), u \in V .
$$

In what follows, we will need the ensuing simple observation.

Proposition 4.1 If $\mathcal{F}_{1}=\left(V_{1}, E_{1}\right)$ is a directed subsemi-tree of $\mathcal{F}$, then $l^{2}\left(V_{1}\right)$ can be regarded as a Hilbert subspace of $l^{2}(V)$. 
Proof It is enough to identify each $f \in l^{2}\left(V_{1}\right)$ with $g \in l^{2}(V)$ defined as $g(v)=f(v)$ for $v \in V_{1}$ and $g(v)=0$ for $v \in V \backslash V_{1}$.

Let us recall the definitions of Segal-Bargmann spaces. For more information on the subject we refer the reader to [2,3] (see also [14] as well as [16-21]).

For $n \in \mathbb{N}$, we set

$$
\langle f, g\rangle_{n}=\int_{\mathbb{C}^{n}} f(z) \overline{g(z)} \mathrm{d} \rho_{n}(z)
$$

for all holomorphic functions $f, g: \mathbb{C}^{n} \rightarrow \mathbb{C}$. Here $\rho_{n}$ denotes the Gaussian measure on $\mathbb{C}^{n}$ defined by

$$
\rho_{n}(\sigma)=\frac{1}{\pi^{n}} \int_{\sigma} \exp \left(-\|z\|_{n}^{2}\right) \mathrm{d} z \quad \text { for each Borel subset } \sigma \text { of } \mathbb{C}^{n},
$$

where for $z:=\left(z_{1}, \ldots, z_{n}\right) \in \mathbb{C}^{n}$,

$$
\|z\|_{n}:=\left(\sum_{i=1}^{n}\left|z_{i}\right|^{2}\right)^{\frac{1}{2}}, \quad \mathrm{~d} z:=\mathrm{d} z_{1} \ldots \mathrm{d} z_{n}
$$

the symbol $\left|z_{i}\right|$ stands for the modulus of $z_{i}, \mathrm{~d} z_{i}=\mathrm{d} x_{i} \mathrm{~d} y_{i}, x_{i}=\operatorname{Re} z_{i}$, and $y_{i}=\operatorname{Im} z_{i}$ $(i=1, \ldots, n)$. Let

$$
B_{n}:=\left\{f: \mathbb{C}^{n} \rightarrow \mathbb{C} \mid f \text { is a holomorphic function and }\langle f, f\rangle_{n}<\infty\right\} .
$$

The Hilbert space $\left(B_{n},\langle\cdot, \cdot\rangle_{n}\right)$ is called the Segal-Bargmann space of an $n$-th order [2]. Set

$$
\varepsilon_{\alpha}(z)=\frac{z^{\alpha}}{\sqrt{\alpha !}}
$$

where $z^{\alpha}:=z_{1}^{\alpha_{1}} \ldots z_{n}^{\alpha_{n}}, \alpha !:=\alpha_{1} ! \ldots \alpha_{n}$ !, for $z=\left(z_{1}, \ldots, z_{n}\right) \in \mathbb{C}^{n}$ and $\alpha=$ $\left(\alpha_{1}, \ldots, \alpha_{n}\right) \in \mathbb{Z}_{+}^{n}$. It can be shown that $\left\{\varepsilon_{\alpha}\right\}_{\alpha \in \mathbb{Z}_{+}^{n}}$ is an orthonormal basis for $B_{n}$ [2].

For $(f)=\left(f_{\alpha}\right)_{\alpha \in V_{n}} \in l^{2}\left(V_{n}\right)$, we define the function $f: \mathbb{C}^{n} \rightarrow \mathbb{C}$ as follows:

$$
f(z):=\sum_{\alpha \in V_{n}} f_{\alpha} \varepsilon_{\alpha}(z), \quad z \in \mathbb{C}^{n}
$$

Then $J_{n}: l^{2}\left(V_{n}\right) \ni(f) \mapsto f \in B_{n}$ is a unitary isomorphism, so we can identify the Segal-Bargmann space $B_{n}$ with the $l^{2}$-Hilbert space defined on vertices of the Bargmann graph $\mathfrak{B}_{n}$.

Next, we recall Bragmann's definition of the Hilbert space of an infinite order [3], which generalizes the definition of $B_{n}$.

In all that follows, the set $\mathbb{Z}_{+}^{n}$ (resp. $\mathbb{C}^{n}$ ) will be interpreted as a subset of $V_{\infty}$ (resp. $l^{2}$, where $l^{2}$ denotes the set of all square-summable complex sequences). Let 
$\varepsilon_{\alpha}(\cdot)$ be defined by the formula (4.2), where $z^{\alpha}:=z_{1}^{\alpha_{1}} \ldots z_{n}^{\alpha_{n}}, \alpha !:=\alpha_{1} ! \ldots \alpha_{n}$ ! for $z=\left(z_{1}, \ldots, z_{n}, \ldots\right) \in l^{2}$ and $\alpha=\left(\alpha_{1}, \ldots, \alpha_{n}, 0,0, \ldots\right) \in V_{\infty}$.

For $(f)=\left(f_{\alpha}\right)_{\alpha \in V_{\infty}} \in l^{2}\left(V_{\infty}\right)$, we define the function $f: l^{2} \rightarrow \mathbb{C}$ as follows:

$$
f(z):=\sum_{\alpha \in V_{\infty}} f_{\alpha} \varepsilon_{\alpha}(z), \quad z \in l^{2} .
$$

The correctness of this definition as well as facts given below were discussed in [17]. Let

$$
B_{\infty}:=\left\{f:(f) \in l^{2}\left(V_{\infty}\right)\right\}
$$

Then $J_{\infty}: l^{2}\left(V_{\infty}\right) \ni(f) \mapsto f \in B_{\infty}$ is linear injection and onto. It is worth mentioning that $J_{\infty}\left(e_{\alpha}\right)=\varepsilon_{\alpha}$ for each $\alpha \in V_{\infty}$. Next, we set

$$
\langle f, g\rangle_{\infty}:=\langle(f),(g)\rangle_{l^{2}\left(V_{\infty}\right)}, \quad(f),(g) \in l^{2}\left(V_{\infty}\right)
$$

Then the mapping $J_{\infty}$ is isometric, and so $\left(B_{\infty},\langle\cdot, \cdot\rangle_{\infty}\right)$ is a Hilbert space, which is called the Segal-Bargmann space of an infinite order. As a consequence, we can identify $B_{\infty}$ with the $l^{2}$-Hilbert space defined on vertices of the Bargmann graph $\mathfrak{B}_{\infty}$.

Remark 4.2 Recall that, in view of Sect. 3 we regard $\mathfrak{B}_{n}(n \in \mathbb{N})$ as a directed subsemi-tree of $\mathfrak{B}_{\infty}$. Hence, by Proposition 4.1, we treat $l^{2}\left(V_{n}\right)$ as a Hilbert subspace of $l^{2}\left(V_{\infty}\right)$. Consequently, by this and the above identifications, $B_{n}$ can be regarded as a Hilbert subspace of $B_{\infty}$ and the restriction of the unitary isomorphism $J_{\infty}$ to $l^{2}\left(V_{n}\right)$ as $J_{n}$. Note also that a Hilbert basis of $B_{\infty}$ is the amalgamation of all bases of $B_{n}$ over all $n \in \mathbb{N}$.

We shall return to Segal-Bargmann spaces in Sect. 6.

\section{Properties of Weighted Shifts on Directed Semi-Trees}

In all that follows, if $A$ is an operator acting in a Hilbert space $\mathcal{H}$, the symbol $\mathcal{D}(A)$ denotes its domain. The graph norm of $A$ is defined as $\|f\|_{A}:=\sqrt{\|f\|^{2}+\|A f\|^{2}}$ for $f \in \mathcal{D}(A)$.

Now suppose that $\mathcal{F}=(V, E)$ is a directed semi-tree and $\operatorname{card}(V) \leq \aleph_{0}$. Let

$$
\lambda:=\left\{\lambda_{(u, v)}:(u, v) \in E\right\}
$$

be an arbitrary subset of $\mathbb{C}$. For $v \in V^{\circ}$, we set

$$
\lambda_{v}=\left\{\lambda_{(u, v)}: u \in \operatorname{Par}(v)\right\} .
$$


In what follows, we also assume that for each $v \in V^{\circ}$,

$$
\left\|\lambda_{v}\right\|:=\left(\sum_{u \in \operatorname{Par}(v)}\left|\lambda_{(u, v)}\right|^{2}\right)^{\frac{1}{2}}<\infty .
$$

The elements of $\lambda$ are called weights.

Note that if $\mathcal{F}$ is a directed tree, then $\lambda_{v}=\left\{\lambda_{(\operatorname{par}(v), v)}\right\}$ for $v \in V^{\circ}$, and so (5.1) is trivially fulfilled. In [9], the symbol $\lambda_{v}$ was used to denote the unique element $\lambda(\operatorname{par}(v), v)$.

For the set of weights $\lambda$, we define the operator $S_{\lambda}$ in $l^{2}(V)$ as follows:

$$
\begin{aligned}
\mathcal{D}\left(S_{\lambda}\right) & =\left\{f \in l^{2}(V): \Lambda_{\mathcal{F}} f \in l^{2}(V)\right\}, \\
S_{\lambda} f & =\Lambda_{\mathcal{F}} f, \quad f \in \mathcal{D}\left(S_{\lambda}\right),
\end{aligned}
$$

where $\Lambda_{\mathcal{F}}$ is the mapping defined on functions $f: V \rightarrow \mathbb{C}$ by

$$
\left(\Lambda_{\mathcal{F}} f\right)(v)= \begin{cases}\sum_{u \in \operatorname{Par}(v)} \lambda_{(u, v)} f(u) & \text { if } v \in V^{\circ} \\ 0 & \text { if } v=\text { root. }\end{cases}
$$

We call the operator $S_{\lambda}$ a weighted shift on a directed semi-tree $\mathcal{F}$. Let us show that the definition of $S_{\lambda}$ is well-posed. The case of a finite $\operatorname{Par}(v)$ is obvious, so let $\operatorname{Par}(v)=\left\{u_{i}: i \in \mathbb{N}\right\}$. Then for $n \leq m(m, n \in \mathbb{N})$, by the Cauchy-Schwartz inequality, we get

$$
\left|\sum_{i=1}^{m} \lambda_{\left(u_{i}, v\right)} f\left(u_{i}\right)-\sum_{i=1}^{n} \lambda_{\left(u_{i}, v\right)} f\left(u_{i}\right)\right| \leq\left(\sum_{i=n}^{m}\left|\lambda_{\left(u_{i}, v\right)}\right|^{2}\right)^{\frac{1}{2}}\left(\sum_{i=n}^{m}\left|f\left(u_{i}\right)\right|^{2}\right)^{\frac{1}{2}} .
$$

From (5.1) and the fact that $f \in l^{2}(V)$ we deduce that

$$
\sum_{i=n}^{m}\left|\lambda_{\left(u_{i}, v\right)}\right|^{2} \rightarrow 0 \text { and } \sum_{i=n}^{m}\left|f\left(u_{i}\right)\right|^{2} \rightarrow 0 \text { as } m, n \rightarrow \infty,
$$

which in connection with the above estimation justifies the correctness of the definition of $S_{\lambda}$.

If $S_{\lambda}$ is a weighted shift on a directed tree, then the above definition of $S_{\lambda}$ coincides with that introduced in [9]. It should be stressed that, in view of Proposition 3.1.10 of [9], the most interesting results for weighted shifts on directed trees can be obtained in the case when $\operatorname{card}(V) \leq \aleph_{0}$. This motivated us to confine our considerations only to this case starting from Sect. 5 .

It turns out that weighted shifts on directed semi-trees have many properties in common with those defined on directed trees. First, we prove the counterpart of Proposition 3.1.2 of [9]. For self-containedness, we include below its proof which goes in a similar fashion as in [9]. 
Proposition 5.1 A weighted shift $S_{\lambda}$ on a directed semi-tree $\mathcal{F}$ is a closed operator.

Proof Suppose that a sequence $\left\{f_{n}\right\}_{n=1}^{\infty}$ of elements from $\mathcal{D}\left(S_{\lambda}\right)$ is convergent to a function $f \in l^{2}(V)$ and the sequence $\left\{S_{\lambda} f_{n}\right\}_{n=1}^{\infty}$ converges to a function $g \in l^{2}(V)$. Take $v \in V$. Then from the reproducing property [see (4.1)] we infer that

$$
\left(S_{\lambda} f_{n}\right)(v)=\left\langle S_{\lambda} f_{n}, e_{v}\right\rangle \rightarrow\left\langle g, e_{v}\right\rangle=g(v) \text { as } n \rightarrow \infty .
$$

We now show that if $v \in V^{\circ}$, then

$$
\left(S_{\lambda} f_{n}\right)(v)=\sum_{u \in \operatorname{Par}(v)} \lambda_{(u, v)} f_{n}(u) \rightarrow \sum_{u \in \operatorname{Par}(v)} \lambda_{(u, v)} f(u)=\left(\Lambda_{\mathcal{F}} f\right)(v)
$$

as $n \rightarrow \infty$. Let $\operatorname{Par}(v)=\left\{u_{i}: i \in \mathbb{N}\right\}$. Then, by the Cauchy-Schwartz inequality, we get

$$
\begin{aligned}
& \left|\sum_{u \in \operatorname{Par}(v)} \lambda_{(u, v)} f_{n}(u)-\sum_{u \in \operatorname{Par}(v)} \lambda_{(u, v)} f(u)\right| \\
& =\lim _{m \rightarrow \infty}\left|\sum_{i=1}^{m} \lambda_{\left(u_{i}, v\right)}\left[f_{n}\left(u_{i}\right)-f\left(u_{i}\right)\right]\right| \\
& \leq \lim _{m \rightarrow \infty}\left(\sum_{i=1}^{m}\left|\lambda_{\left(u_{i}, v\right)}\right|^{2}\right)^{\frac{1}{2}}\left(\sum_{i=1}^{m}\left|\left(f_{n}-f\right)\left(u_{i}\right)\right|^{2}\right)^{\frac{1}{2}} \\
& \leq\left\|\lambda_{v}\right\|\left\|f_{n}-f\right\| \rightarrow 0 \text { as } n \rightarrow \infty,
\end{aligned}
$$

which proves (5.4). Next, since $\left(S_{\lambda} f_{n}\right)$ (root) $=0$ by (5.2), we deduce from (5.3) that $g$ (root) $=0$. We conclude that $\left(\Lambda_{\mathcal{F}} f\right)(v)=g(v)$ for each $v \in V$. Hence $\Lambda_{\mathcal{F}} f \in l^{2}(V)$, and so $f \in \mathcal{D}\left(S_{\lambda}\right)$ and $g=S_{\lambda} f$. The proof is complete.

We now prove Theorem 5.2 which in combination with Theorem 7.4 may be regarded as an adaptation of Proposition 3.1.3 of [9] to the case of directed semitrees. It should however be pointed out that the last-mentioned result does not transfer automatically to our context and the proofs (in particular, that of Theorem 7.4) require some new methods. In what follows, we maintain the conventions of [9] that $0 \cdot \infty=0$ and that the sum over an empty set is equal zero.

Theorem 5.2 Let $S_{\lambda}$ be a weighted shift on a directed semi-tree $\mathcal{F}=(V, E)$ with weights $\lambda=\left\{\lambda_{(u, v)}:(u, v) \in E\right\}$. Then the following assertions hold:

$$
\mathcal{D}\left(S_{\lambda}\right)=\left\{f \in l^{2}(V): \sum_{v \in V^{\circ}}\left|\sum_{u \in \operatorname{Par}(v)} \lambda_{(u, v)} f(u)\right|^{2}<\infty\right\}
$$

and 
(ii)

$$
S_{\lambda} f=\sum_{v \in V^{\circ}}\left(\sum_{u \in \operatorname{Par}(v)} \lambda_{(u, v)} f(u)\right) e_{v}, \quad f \in \mathcal{D}\left(S_{\lambda}\right) .
$$

$$
\|f\|_{S_{\lambda}}^{2} \leq \sum_{u \in V}\left(1+\sum_{v \in \operatorname{Chi}(u)}\left\|\lambda_{v}\right\|^{2}\right)|f(u)|^{2}, \quad f \in \mathcal{D}\left(S_{\lambda}\right),
$$

where $\|\cdot\|_{S_{\lambda}}$ denotes the graph norm of the operator $S_{\lambda}$;

(iii) if $u \in V$, then:

(a) $e_{u} \in \mathcal{D}\left(S_{\lambda}\right)$ if and only if $\sum_{v \in \operatorname{Chi}(u)}\left|\lambda_{(u, v)}\right|^{2}<\infty$,

(b) if $e_{u} \in \mathcal{D}\left(S_{\lambda}\right)$, then

$$
S_{\lambda} e_{u}=\sum_{v \in \operatorname{Chi}(u)} \lambda_{(u, v)} e_{v} \text { and }\left\|S_{\lambda} e_{u}\right\|^{2}=\sum_{v \in \operatorname{Chi}(u)}\left|\lambda_{(u, v)}\right|^{2} ;
$$

(iv) if $W$ be a subset of $V$ such that $\operatorname{Par}(v) \cap W=\emptyset$ or $\operatorname{Par}(v) \subset W$ for each $v \in V$, then $f \cdot \chi_{W} \in \mathcal{D}\left(S_{\lambda}\right)$ for each $f \in \mathcal{D}\left(S_{\lambda}\right)$, where $\chi_{W}$ denotes the characteristic function of $W$;

(v) if $\left\{e_{v}\right\}_{v \in V} \subset \mathcal{D}\left(S_{\lambda}\right)$, then $S_{\lambda}$ is densely defined.

Proof If $f \in \mathcal{D}\left(S_{\lambda}\right)$, then $\Lambda_{\mathcal{F}} f \in l^{2}(V)$. By this and (5.2), we have

$$
\infty>\left\|\Lambda_{\mathcal{F}} f\right\|^{2}=\sum_{v \in V}\left|\left(\Lambda_{\mathcal{F}} f\right)(v)\right|^{2}=\sum_{v \in V^{\circ}}\left|\sum_{u \in \operatorname{Par}(v)} \lambda_{(u, v)} f(u)\right|^{2},
$$

which gives (i).

(ii) For $f \in \mathcal{D}\left(S_{\lambda}\right)$, we obtain

$$
\begin{aligned}
\|f\|_{S_{\lambda}}^{2} & =\|f\|^{2}+\left\|S_{\lambda} f\right\|^{2}=\sum_{v \in V}|f(v)|^{2}+\sum_{v \in V^{\circ}}\left|\sum_{u \in \operatorname{Par}(v)} \lambda_{(u, v)} f(u)\right|^{2} \\
& \stackrel{(1)}{\leq} \sum_{v \in V}|f(v)|^{2}+\sum_{v \in V^{\circ}}\left[\left(\sum_{u \in \operatorname{Par}(v)}\left|\lambda_{(u, v)}\right|^{2}\right)\left(\sum_{u \in \operatorname{Par}(v)}|f(u)|^{2}\right)\right] \\
& =\sum_{v \in V}|f(v)|^{2}+\sum_{v \in V^{\circ}} \sum_{u \in \operatorname{Par}(v)}\left\|\lambda_{v}\right\|^{2}|f(u)|^{2} \\
& \stackrel{(2)}{=} \sum_{v \in V}|f(v)|^{2}+\sum_{u \in V} \sum_{v \in \operatorname{Chi}(u)}\left\|\lambda_{v}\right\|^{2}|f(u)|^{2} \\
& =\sum_{u \in V}|f(u)|^{2}+\sum_{u \in V}\left(\sum_{v \in \operatorname{Chi}(u)}\left\|\lambda_{v}\right\|^{2}\right)|f(u)|^{2} \\
& =\sum_{u \in V}\left(1+\sum_{v \in \operatorname{Chi}(u)}\left\|\lambda_{v}\right\|^{2}\right)|f(u)|^{2},
\end{aligned}
$$


where (1) results from a combination of (5.1), the fact that $f \in l^{2}(V)$ and the CauchySchwartz inequality, whereas (2) is a consequence of the equality

$$
\bigcup_{v \in V^{\circ}}\{(u, v):(u, v) \in E\}=\bigcup_{u \in V}\{(u, v):(u, v) \in E\} .
$$

(iii) Take $u \in V$ and observe that

$$
\sum_{v \in V^{\circ}}\left|\sum_{w \in \operatorname{Par}(v)} \lambda_{(w, v)} e_{u}(w)\right|^{2}=\sum_{\left\{v \in V^{\circ}: u \in \operatorname{Par}(v)\right\}}\left|\lambda_{(u, v)}\right|^{2}=\sum_{v \in \operatorname{Chi}(u)}\left|\lambda_{(u, v)}\right|^{2} .
$$

In view of (i), $e_{u} \in \mathcal{D}\left(S_{\lambda}\right)$ if and only if the above sum is finite, which implies (a). Furthermore, take $u \in V$ and observe that for $w \in V^{\circ}$, we get

$$
\begin{aligned}
\left(S_{\lambda} e_{u}\right)(w) \stackrel{(5.2)}{=} \sum_{\kappa \in \operatorname{Par}(w)} \lambda_{(\kappa, w)} e_{u}(\kappa) \\
= \begin{cases}\lambda_{(u, w)} & \text { if } u \in \operatorname{Par}(w) \text { iff } w \in \operatorname{Chi}(u)) \\
0 & \text { otherwise }\end{cases} \\
=\left(\sum_{v \in \operatorname{Chi}(u)} \lambda_{(u, v)} e_{v}\right)(w) .
\end{aligned}
$$

If $w=$ root, then again owing to (5.2) we get $\left(S_{\lambda} e_{u}\right)($ root $)=0$, and

$$
\left(\sum_{v \in \operatorname{Chi}(u)} \lambda_{(u, v)} e_{v}\right)(\text { root })=\sum_{v \in \operatorname{Chi}(u)} \lambda_{(u, v)} e_{v}(\text { root })=0,
$$

because root $\notin \operatorname{Chi}(u)$. This implies (b).

(iv) Since, for $v \in V^{\circ}$, we have

$$
\sum_{u \in \operatorname{Par}(v)} \lambda_{(u, v)}\left(f \cdot \chi_{W}\right)(u) \stackrel{(5.2)}{=} \begin{cases}\sum_{u \in \operatorname{Par}(v)} \lambda_{(u, v)} f(u) & \text { if } \operatorname{Par}(v) \subset W, \\ 0 & \text { if } \operatorname{Par}(v) \cap W=\emptyset,\end{cases}
$$

it follows that

$$
\sum_{v \in V^{\circ}}\left|\sum_{u \in \operatorname{Par}(v)} \lambda_{(u, v)}\left(f \cdot \chi_{W}\right)(u)\right|^{2} \leq \sum_{v \in V^{\circ}}\left|\sum_{u \in \operatorname{Par}(v)} \lambda_{(u, v)} f(u)\right|^{2} .
$$

The latter sum is finite, because $f \in \mathcal{D}\left(S_{\lambda}\right)$ and (i) holds. Thus, again in view of (i), we deduce that $f \cdot \chi_{W} \in \mathcal{D}\left(S_{\lambda}\right)$.

(v) is clear, because $\left\{e_{u}\right\}_{u \in V}$ is an orthonormal basis of $l^{2}(V)$.

For the sake of completeness, we state a simple observation. 
Proposition 5.3 If $\lambda$ is a set of weights and $S_{\lambda}$ is an operator in $l^{2}(V)$ defined by the formula given in Theorem 5.2 (i), then $S_{\lambda}$ is a weighted shift on a directed semi-tree $\mathcal{F}$.

Now let us turn to condition (v) of Theorem 5.2 and consider the following problem.

Problem 5.4 Does the inclusion $\left\{e_{v}\right\}_{v \in V} \subset \mathcal{D}\left(S_{\lambda}\right)$ hold if $S_{\lambda}$ is a densely defined weighted shift on a directed semi-tree?

As condition ( $v$ ) of Proposition 3.1.3 of [9] shows, the answer is in the affirmative in the case of weighted shifts on directed trees. We remain the above question in its full generality unanswered. Nevertheless, we can give a patrial solution to this problem. The inspiration for its proof comes from the last-mentioned result from [9].

Proposition 5.5 Let $S_{\lambda}$ be as in Theorem 5.2 and

$$
\sum_{v \in \operatorname{Chi}(u)}\left(\sum_{w \in \operatorname{Par}(v) \backslash\{u\}}\left|\lambda_{(w, v)}\right|^{2}\right)<\infty \text { for each } u \in V .
$$

Then the operator $S_{\lambda}$ is densely defined if and only if $\left\{e_{v}\right\}_{v \in V} \subset \mathcal{D}\left(S_{\lambda}\right)$.

Proof The only implication that requires care is $(\Rightarrow)$. Let $S_{\lambda}$ be densely defined. Suppose on the contrary that there exists $u \in V$ such that $e_{u} \notin \mathcal{D}\left(S_{\lambda}\right)$. Then, by condition (iii a) of Theorem 5.2, we have

$$
\sum_{v \in \operatorname{Chi}(u)}\left|\lambda_{(u, v)}\right|^{2}=\infty
$$

For a fixed $f \in \mathcal{D}\left(S_{\lambda}\right)$, we get

$$
\sum_{v \in \operatorname{Chi}(u)}\left|\lambda_{(u, v)} f(u)+\sum_{w \in \operatorname{Par}(v) \backslash\{u\}} \lambda_{(w, v)} f(w)\right|^{2}<\infty,
$$

because the above sum can be estimated by $\sum_{v \in V^{\circ}}\left|\sum_{w \in \operatorname{Par}(v)} \lambda_{(w, v)} f(w)\right|^{2}$ which is finite by Theorem 5.2 (i). Next, observe that we also have

$$
\sum_{v \in \operatorname{Chi}(u)}\left|\sum_{w \in \operatorname{Par}(v) \backslash\{u\}} \lambda_{(w, v)} f(w)\right|^{2}<\infty .
$$

Indeed, by the Cauchy-Schwartz inequality, this sum is bounded above by

$$
\|f\|^{2} \sum_{v \in \operatorname{Chi}(u)}\left(\sum_{w \in \operatorname{Par}(v) \backslash\{u\}}\left|\lambda_{(w, v)}\right|^{2}\right)
$$


and in view of (5.6) the latter expression is finite. A combination of (5.8), (5.7) and (5.9) leads to the conclusion that $\left\langle f, e_{u}\right\rangle=f(u)=0$. Hence each $f \in \mathcal{D}\left(S_{\lambda}\right)$ is orthogonal to $e_{u}$, but the operator $S_{\lambda}$ is densely defined, so we arrive at a contradiction.

It is worth mentioning that condition (5.6) is fulfilled for a directed semi-tree $\mathcal{F}=$ $(V, E)$ such that

$$
\operatorname{card}\{v \in \operatorname{Chi}(u): \operatorname{card}(\operatorname{Par}(v))>1\}<\aleph_{0} \text { for each } u \in V
$$

A look at Example 5.7 given below reveals that (5.6) is not necessary for the inclusion $\left\{e_{v}\right\}_{v \in V} \subset \mathcal{D}\left(S_{\lambda}\right)$ to hold (consider $u=v_{0}$ therein).

Remark 5.6 Regarding condition (iii a) of Theorem 5.2, if card $(\mathrm{Chi}(u))<\aleph_{0}$ for each $u \in V$, then we clearly get $\left\{e_{v}\right\}_{v \in V} \subset \mathcal{D}\left(S_{\lambda}\right)$. It worth noting that, in view of Remark 3.4 , such situation holds for each weighted shift acting on the $n$-finite Bargmann graph $\mathfrak{B}_{n}(n \in \mathbb{N})$.

Next, note that if $\mathcal{F}$ is a directed tree, then the inequality in Theorem 5.2 (ii) turns into the equality taking a considerably simplified form as in Proposition 3.1.3 (ii) of [9]. That equality was used in [9] to show that

$$
S_{\lambda}=\overline{S_{\lambda} \mid \mathcal{E}_{V}},
$$

where $\mathcal{E}_{V}$ is the linear span of the set $\left\{e_{v}: v \in V\right\}$, if the operator $S_{\lambda}$ is densely defined. In the case of weighted shifts on directed semi-trees we cannot employ an analogous argument to that of [9] to obtain (5.10). This is because the right-hand side of the inequality in Theorem 5.2 (ii) may be infinite if a directed semi-tree has a countable set of vertices, as evidenced in Example 5.7. It is also worth noting that the considered inequality may be strict even if its right-hand side is finite, which was shown in Example 5.8. We will return to the discussion on (5.10) in Sect. 7.

Example 5.7 Take $\mathcal{F}=(V, E)$ such that

$$
V=\left\{\text { root, } v_{0}\right\} \cup\left\{v_{n}: n \in \mathbb{N}\right\} \cup\left\{v_{(m, n)}: m, n \in \mathbb{N}\right\}
$$

and

$$
\begin{aligned}
E= & \left\{\left(\text { root }, v_{0}\right)\right\} \cup\left\{\left(\text { root }, v_{(m, n)}\right): m, n \in \mathbb{N}\right\} \cup\left\{\left(v_{0}, v_{n}\right): n \in \mathbb{N}\right\} \\
& \cup\left\{\left(v_{(m, n)}, v_{n}\right): m, n \in \mathbb{N}\right\} .
\end{aligned}
$$

For $m, n \in \mathbb{N}$, define weights $\lambda$ as follows:

$$
\lambda_{\left(\text {root }, v_{0}\right)}=\lambda_{\left(\operatorname{root}, v_{(m, n)}\right)}=0, \quad \lambda_{\left(v_{0}, v_{n}\right)}=\frac{1}{n}, \quad \lambda_{\left(v_{(m, n)}, v_{n}\right)}=\frac{1}{\sqrt{2^{m} n}} .
$$


If $u=v_{0}$, then

$$
\begin{aligned}
\sum_{v \in \operatorname{Chi}(u)}\left\|\lambda_{v}\right\|^{2} & =\sum_{n=1}^{\infty}\left\|\lambda_{v_{n}}\right\|^{2}=\sum_{n=1}^{\infty}\left(\left|\lambda_{\left(v_{0}, v_{n}\right)}\right|^{2}+\sum_{m=1}^{\infty}\left|\lambda_{\left(v_{(m, n)}, v_{n}\right)}\right|^{2}\right) \\
& =\sum_{n=1}^{\infty}\left(\frac{1}{n^{2}}+\sum_{m=1}^{\infty} \frac{1}{2^{m} n}\right)=\sum_{n=1}^{\infty}\left(\frac{1}{n^{2}}+\frac{1}{n}\right)=\infty
\end{aligned}
$$

A moment's thought reveals that, in view of condition (iii a) of Theorem 5.2, $e_{v} \in \mathcal{D}\left(S_{\lambda}\right)$ for each $v \in V$. Now it suffices to take $f \in \mathcal{D}\left(S_{\lambda}\right)$ such that $f(u) \neq 0$ (e.g. $f=e_{u}$ ) to see that the right-hand side of the inequality in Theorem 5.2 (ii) is infinite. Remark additionally that, by (v) of Theorem 5.2, the operator $S_{\lambda}$ is densely defined.

Example 5.8 Let us consider $\mathcal{F}=(V, E)$, where

$$
V=\left\{v_{1}, v_{2}, v_{3}, v_{4}\right\}, \quad E=\left\{\left(v_{1}, v_{2}\right),\left(v_{1}, v_{3}\right),\left(v_{2}, v_{4}\right),\left(v_{3}, v_{4}\right)\right\}
$$

Let $S_{\lambda}$ be a weighted shift with weights $\lambda$ such that $\lambda_{\left(v_{2}, v_{4}\right)}=-\lambda_{\left(v_{3}, v_{4}\right)} \neq 0$. Take a constant function $f=1$ on $V$. Then

$$
\|f\|_{S_{\lambda}}^{2}=4+\left|\lambda_{\left(v_{1}, v_{2}\right)}\right|^{2}+\left|\lambda_{\left(v_{1}, v_{3}\right)}\right|^{2},
$$

whereas the right-hand side of (ii) takes the form

$$
4+\left|\lambda_{\left(v_{1}, v_{2}\right)}\right|^{2}+\left|\lambda_{\left(v_{1}, v_{3}\right)}\right|^{2}+4\left|\lambda_{\left(v_{2}, v_{4}\right)}\right|^{2} .
$$

Remark 5.9 It is also worth mentioning that for a weighted shift $S_{\lambda}$ on a directed tree $\mathcal{F}=(V, E)$ the description of $\mathcal{D}\left(S_{\lambda}\right)$ in Theorem 5.2 reduces to that given in [9, Proposition 3.1.3]. Indeed, then each $v \in V^{\circ}$ has a unique parent $\operatorname{par}(v)$. If we denote $\lambda(\operatorname{par}(v), v)$ by $\widehat{\lambda}_{v}$, we get

$$
\begin{aligned}
\sum_{v \in V^{\circ}}\left|\sum_{u \in \operatorname{Par}(v)} \lambda_{(u, v)} f(u)\right|^{2} & =\sum_{v \in V^{\circ}}\left|\widehat{\lambda}_{v} f(\operatorname{par}(v))\right|^{2} \\
& =\sum_{u \in V}\left(\sum_{v \in \operatorname{Chi}(u)}\left|\widehat{\lambda}_{v}\right|^{2}\right)|f(u)|^{2}, \quad f \in \mathcal{D}\left(S_{\lambda}\right),
\end{aligned}
$$

where the last equality results from Proposition 2.3.

We close this section by showing that the assumption that $\operatorname{Par}(v) \cap W=\emptyset$ or $\operatorname{Par}(v) \subset W$ for each $v \in V$ in Theorem 5.2 (iv) cannot be dropped without affecting the validity of the result. 
Example 5.10 To see this, consider a directed semi-tree $\mathcal{F}=(V, E)$ such that

$$
\begin{aligned}
& V=\left\{v_{0}, v_{(n, 1)}, v_{(n, 2)}: n \in \mathbb{N}\right\}, \\
& E=\left\{\left(v_{0}, v_{(1,1)}\right),\left(v_{0}, v_{(1,2)}\right)\right\} \cup\left\{\left(v_{(n, k)}, v_{(n+1, j)}\right): k, j \in\{1,2\}, n \in \mathbb{N}\right\},
\end{aligned}
$$

and define a weighted shift $S_{\lambda}$ with weights $\lambda$ given as follows:

$$
\begin{aligned}
\lambda_{\left(v_{0}, v_{(1,1)}\right)} & =\lambda_{\left(v_{0}, v_{(1,2)}\right)}=1, \\
\lambda_{\left(v_{(n, 1)}, v_{(n+1,1)}\right)} & =\lambda_{\left(v_{(n, 2)}, v_{(n+1,2)}\right)}=n, \quad n \in \mathbb{N}, \\
\lambda_{\left(v_{(n, 1)}, v_{(n+1,2)}\right)} & =\lambda_{\left(v_{(n, 2)}, v_{(n+1,1)}\right)}=-n, \quad n \in \mathbb{N} .
\end{aligned}
$$

Note that each vertex $v \in V^{0}$ has at most two parents, so (5.1) holds. Take a function $f: V \rightarrow \mathbb{C}$ such that

$$
f\left(v_{0}\right)=1 \text { and } f\left(v_{(n, k)}\right)=\frac{1}{n} \quad \text { for all } k \in\{1,2\}, n \in \mathbb{N} \text {. }
$$

Then $\sum_{v \in V}|f(v)|^{2}=2\left(\sum_{n=1}^{\infty} \frac{1}{n^{2}}\right)+1<\infty$, and so $f \in l^{2}(V)$. Moreover,

$$
\sum_{u \in \operatorname{Par}(v)} \lambda_{(u, v)} f(u)=0
$$

for $v \in V^{0} \backslash\left\{v_{(1,1)}, v_{(1,2)}\right\}$. If in turn $v \in\left\{v_{(1,1)}, v_{(1,2)}\right\}$, then this sum is equal to one. As a consequence, we get

$$
\sum_{v \in V^{\circ}}\left|\sum_{u \in \operatorname{Par}(v)} \lambda_{(u, v)} f(u)\right|^{2}=2<\infty .
$$

Therefore, $f \in \mathcal{D}\left(S_{\lambda}\right)$. If we set $W=\left\{v_{(n, 2)}: n \in \mathbb{N}\right\}$, then the sum

$$
\sum_{v \in V^{\circ}}\left|\sum_{u \in \operatorname{Par}(v)} \lambda_{(u, v)}\left(f \cdot \chi_{W}\right)(u)\right|^{2}
$$

can be written as

$$
\begin{aligned}
& \left|\lambda_{\left(v_{(1,2)}, v_{(2,1)}\right)} f\left(v_{(1,2)}\right)\right|^{2}+\left|\lambda_{\left(v_{(1,2)}, v_{(2,2)}\right)} f\left(v_{(1,2)}\right)\right|^{2} \\
& \quad+\left|\lambda_{\left(v_{(2,2)}, v_{(3,1)}\right)} f\left(v_{(2,2)}\right)\right|^{2}+\left|\lambda_{\left.\left(v_{(2,2)}\right), v_{(3,2)}\right)} f\left(v_{(2,2)}\right)\right|^{2}+\ldots,
\end{aligned}
$$

where each summand equals one. Thus $f \cdot \chi_{W}$ does not belong to $\mathcal{D}\left(S_{\lambda}\right)$. 


\section{Generalized Creation Operators as Weighted Shifts on Directed Semi-Trees}

In [3], Bargmann defined the generalized creation operator in $B_{\infty}$ in a direction $a \in l^{2}$ by

$$
\begin{aligned}
\mathcal{D}\left(A_{a}^{+}\right) & =\left\{f \in B_{\infty}:\langle\cdot, a\rangle f \in B_{\infty}\right\}, \\
\left(A_{a}^{+} f\right)(z) & =\langle z, a\rangle f(z), \quad f \in \mathcal{D}\left(A_{a}^{+}\right), z \in l^{2},
\end{aligned}
$$

where $\langle\cdot,-\rangle$ is the standard inner product in $l^{2}$. Such operators as well as, related with them, Bargmann's generalized anihilation operators (see [3]) were investigated in [17], where in particular was shown that each generalized creation operator in $B_{\infty}$ is subnormal. An incorrect formula used in the proof of this result was recently improved and justified by the author in [22].

Our aim is to show that each operator $A_{a}^{+}$is unitarily isomorphic to a weighted shift on the infinite Bargmann directed semi-tree.

Recall that for each $(f)=\left(f_{\alpha}\right)_{\alpha \in V_{\infty}} \in l^{2}\left(V_{\infty}\right)$ there exists a unique $f \in B_{\infty}$ such that $f=J_{\infty}[(f)]$, where $J_{\infty}$ is a unitary isomorphism introduced in Sect. 4. Fix a direction $a=\left(a_{1}, a_{2}, \ldots\right) \in l^{2}$. Proposition 3 of [17], which plays a crucial role in our further consideration, says that for $(f) \in l^{2}\left(V_{\infty}\right)$,

$$
f \in \mathcal{D}\left(A_{a}^{+}\right) \text {if and only if } \sum_{\beta \in V_{\infty}}\left|\sum_{i \in \mathbb{N}} f_{\beta-\delta_{i}} \overline{a_{i}} \sqrt{\beta_{i}}\right|^{2}<\infty,
$$

where $f_{\beta-\delta_{i}}:=0$ if $\beta_{i}=0(i \in \mathbb{N})$, and

$$
A_{a}^{+} f=\sum_{\beta \in V_{\infty}}\left(\sum_{i \in \mathbb{N}} f_{\beta-\delta_{i}} \overline{a_{i}} \sqrt{\beta_{i}}\right) \varepsilon_{\beta} \text { if } f \in \mathcal{D}\left(A_{a}^{+}\right) .
$$

Here and in all that follows, $\varepsilon_{\beta}$ is an element of the Hilbert basis in $B_{\infty}$ given by (4.2), and $\beta_{i}$ stands for the $i$-th term of $\beta \in V_{\infty}$.

Next, define the operator $\mathcal{A}_{a}^{+}$in $l^{2}\left(V_{\infty}\right)$ as

$$
\mathcal{A}_{a}^{+}=J_{\infty}^{-1} \circ A_{a}^{+} \circ J_{\infty}
$$

Remark 6.1 If $a \in \mathbb{C}^{n}$, then the domain and the image of $A_{a}^{+}$are included in $B_{n}$, so $A_{a}^{+}$can be regarded as an operator acting in $B_{n}$ and $\mathcal{A}_{a}^{+}=J_{n}^{-1} \circ A_{a}^{+} \circ J_{n}$ (consult Remark 4.2). Note that then $\mathcal{A}_{a}^{+}$acts in $l^{2}\left(V_{n}\right)$.

In the rest of the paper, we confine our attention to the case when $a \in l^{2}$, but our results can be easily reformulated for $a \in \mathbb{C}^{n}$. If it is necessary, we will highlight the difference between those two cases.

We are in a position to prove the main results of this section.

Theorem 6.2 For each $a \in l^{2}$ the operator $\mathcal{A}_{a}^{+}$is a weighted shift on the infinite Bargmann semi-tree $\mathfrak{B}_{\infty}$. 
Proof Take $a=\left(a_{1}, a_{2}, \ldots\right) \in l^{2}$. In view of $(6.5),(f) \in \mathcal{D}\left(\mathcal{A}_{a}^{+}\right)$if and only if $f:=J_{\infty}[(f)] \in \mathcal{D}\left(A_{a}^{+}\right)$. If this is the case, then the sum in (6.3) is finite. We can rewrite it, using on the way Lemma 3.3 (iii), as follows:

$$
\begin{aligned}
\sum_{\beta \in V_{\infty}}\left|\sum_{i \in \mathbb{N}} f_{\beta-\delta_{i}} \overline{a_{i}} \sqrt{\beta_{i}}\right|^{2} & =\sum_{\beta \in V_{\infty}^{\circ}}\left|\sum_{\alpha \in \operatorname{Par}(\beta)} f_{\alpha} \overline{a_{i(\alpha, \beta)}} \sqrt{\beta_{i(\alpha, \beta)}}\right|^{2} \\
& =\sum_{\beta \in V_{\infty}^{\circ}}\left|\sum_{\alpha \in \operatorname{Par}(\beta)} \lambda_{(\alpha, \beta)} f_{\alpha}\right|^{2},
\end{aligned}
$$

where

$$
\lambda_{(\alpha, \beta)}:=\overline{a_{i(\alpha, \beta)}} \sqrt{\beta_{i(\alpha, \beta)}}
$$

and $i(\alpha, \beta)$ is a unique natural number such that $\alpha+\delta_{i(\alpha, \beta)}=\beta$, or equivalently $\alpha_{i(\alpha, \beta)}+1=\beta_{i(\alpha, \beta)}$. Consequently,

$$
\mathcal{D}\left(\mathcal{A}_{a}^{+}\right)=\left\{(f) \in l^{2}\left(V_{\infty}\right): \sum_{\beta \in V_{\infty}^{\circ}}\left|\sum_{\alpha \in \operatorname{Par}(\beta)} \lambda_{(\alpha, \beta)} f_{\alpha}\right|^{2}<\infty\right\} .
$$

It also should be noted that

$$
\sum_{\alpha \in \operatorname{Par}(\beta)}\left|\lambda_{(\alpha, \beta)}\right|^{2}=\sum_{i=1}^{\infty}\left|a_{i}\right|^{2}\left|\beta_{i}\right|<\infty \text { for each } \beta \in V_{\infty}^{\circ}
$$

because all but a finite number of terms $\beta_{i}$ equal zero.

Next, for $(f) \in \mathcal{D}\left(\mathcal{A}_{a}^{+}\right)$, using (6.4) and performing similar calculations as in (6.6), we get

$$
A_{a}^{+}\left(J_{\infty}[(f)]\right)=\sum_{\beta \in V_{\infty}}\left(\sum_{i \in \mathbb{N}} f_{\beta-\delta_{i}} \overline{a_{i}} \sqrt{\beta_{i}}\right) \varepsilon_{\beta}=\sum_{\beta \in V_{\infty}^{\circ}}\left(\sum_{\alpha \in \operatorname{Par}(\beta)} \lambda_{(\alpha, \beta)} f_{\alpha}\right) \varepsilon_{\beta} .
$$

From (6.5), this and the fact that $J_{\infty}^{-1}\left(\varepsilon_{\beta}\right)=e_{\beta}$ for each $\beta \in V_{\infty}$ we infer that

$$
\mathcal{A}_{a}^{+}[(f)]=J_{\infty}^{-1}\left(A_{a}^{+}\left(J_{\infty}[(f)]\right)\right)=\sum_{\beta \in V_{\infty}^{\circ}}\left(\sum_{\alpha \in \operatorname{Par}(\beta)} \lambda_{(\alpha, \beta)} f_{\alpha}\right) e_{\beta} .
$$

Owing to the conditions (6.9), (6.8) and (6.10), we can call upon Proposition 5.3 to deduce that $\mathcal{A}_{a}^{+}$is a weighted shift on $\mathfrak{B}_{\infty}$.

We know, by Remark 5.6, that $\left\{e_{\alpha}\right\}_{\alpha \in V_{n}} \subset \mathcal{D}\left(\mathcal{A}_{a}^{+}\right)$if $a \in \mathbb{C}^{n}$. It turns out that this result can be obtained in a more general case when $a \in l^{2}$. However, to show this we cannot apply Proposition 5.5, because (as is easily checked) $\mathcal{A}_{a}^{+}$does not satisfy condition (5.6). 
Proposition 6.3 For each $a \in l^{2}$ we have $\left\{e_{\alpha}\right\}_{\alpha \in V_{\infty}} \subset \mathcal{D}\left(\mathcal{A}_{a}^{+}\right)$.

Proof Take $a=\left(a_{1}, a_{2}, \ldots\right) \in l^{2}$. By Theorem 6.2, $\mathcal{A}_{a}^{+}$is a weighted shift on $\mathfrak{B}_{\infty}$. Let $\lambda=\left\{\lambda_{(\alpha, \beta)}:(\alpha, \beta) \in E_{\infty}\right\}$ be the set of its weights which are given by (6.7). Then for $\alpha=\left(\alpha_{1}, \ldots, \alpha_{m}, 0,0, \ldots\right) \in V_{\infty}$, by Lemma 3.3 (i), we have

$$
\begin{aligned}
\sum_{\beta \in \operatorname{Chi}(\alpha)}\left|\lambda_{(\alpha, \beta)}\right|^{2} & =\sum_{i \in \mathbb{N}}\left|\lambda_{\left(\alpha, \alpha+\delta_{i}\right)}\right|^{2}=\sum_{i \in \mathbb{N}}\left|\overline{a_{i}} \sqrt{\alpha_{i}+1}\right|^{2} \\
& =\sum_{i=1}^{m}\left|\overline{a_{i}} \sqrt{\alpha_{i}+1}\right|^{2}+\sum_{i=m+1}^{\infty}\left|a_{i}\right|^{2}<\infty .
\end{aligned}
$$

The right-hand side of the above expression is finite, because $a \in l^{2}$. This, by the condition (iii) of Theorem 5.2, implies that $e_{\alpha} \in \mathcal{D}\left(\mathcal{A}_{a}^{+}\right)$for each $\alpha \in V_{\infty}$.

From Proposition 6.3 and Theorem 5.2 (v) we immediately obtain the following corollary.

Corollary 6.4 For each $a \in l^{2}$ the operator $\mathcal{A}_{a}^{+}$is densely defined.

Finally, note that, since the operators $\mathcal{A}_{a}^{+}$and $A_{a}^{+}$are unitarily isomorphic, the density of $\mathcal{D}\left(\mathcal{A}_{a}^{+}\right)$in $l^{2}\left(V_{\infty}\right)$ implies the density of $\mathcal{D}\left(A_{a}^{+}\right)$in $B_{\infty}$.

\section{A Core of a Weighted Shift on a Directed Semi-Tree}

In this section we discuss another question which naturally arises from comparison of Proposition 3.1.3 of [9] with Theorem 5.2.

Problem 7.1 Does $S_{\lambda}=\overline{S_{\lambda} \mid \mathcal{E}_{V}}$ for a weighted shift $S_{\lambda}$ on a directed semi-tree, where $\mathcal{E}_{V}$ is the linear span of the set $\left\{e_{v}: v \in V\right\}$ ?

As already mentioned in Sect. 5, the answer is in the affirmative for weighted shifts on directed trees. In what follows, we shall formulate some sufficient conditions for the above equality to hold in a general case.

Let $\mathcal{F}=(V, E)$ be a directed semi-tree, where $\operatorname{card}(V) \leq \aleph_{0}$. Consider a sequence $\left\{S_{n}\right\}_{n=1}^{\infty}$ of subsets of $V$ such that:

(1) $S_{n} \subset S_{n+1}$ for each $n \in \mathbb{N}$,

(2) $V=\bigcup_{n=1}^{\infty} S_{n}$,

(3) $V=\bigcup_{n=1}^{\infty} \operatorname{Par}\left(S_{n}\right)$,

(4) $\operatorname{card}\left(\operatorname{Par}\left(S_{n}\right)\right)<\aleph_{0}$ for each $n \in \mathbb{N}$,

It is easily seen that such a sequence $\left\{S_{n}\right\}_{n=1}^{\infty}$ can always be constructed for $\mathcal{F}$.

Remark 7.2 The following relations between the above conditions can be observed. The implication (2) $\Rightarrow(3)$ is not true in general, as evidenced in Example 7.3 (a). However, if we additionally assume that $\operatorname{Chi}(v) \neq \varnothing$ for each $v \in V$, then (3) results from (2). Indeed, take $v \in V$ and $u \in \operatorname{Chi}(v)$. In view of (2), $u$ belongs to $S_{n}$ for some 
$n \in \mathbb{N}$, which means that $v \in \operatorname{Par}\left(S_{n}\right)$. This proves (3). Next, note that the implication $(3) \Rightarrow(2)$ does not hold even if conditions (1) and (4) are satisfied. To see this consult Example 7.3 (b). Further, it is clear that (1) implies that $\operatorname{Par}\left(S_{n}\right) \subset \operatorname{Par}\left(S_{n+1}\right)$ for each $n \in \mathbb{N}$, but it can be shown that the converse statement is not true even if (2) and (3) hold. Finally, remark that all $S_{n}$ 's which satisfy (1)-(4) may be countable. Here we refer the reader to Example 7.3 (c).

Example 7.3 (a) Let $\mathcal{F}=(V, E)$ and $S_{n}$ 's be such that

$$
\begin{aligned}
V & =\{v\} \cup\left\{v_{k}: k \in \mathbb{Z}_{+}\right\}, \\
E & =\left\{\left(v_{0}, v\right)\right\} \cup\left\{\left(v_{k}, v_{k+1}\right): k \in \mathbb{Z}_{+}\right\}, \\
S_{n} & =\left\{v, v_{0}, \ldots, v_{n}\right\}, \quad n \in \mathbb{N}
\end{aligned}
$$

(see Fig. 3a). Then (2) holds, but (3) does not, because $\bigcup_{n=1}^{\infty} \operatorname{Par}\left(S_{n}\right)=V \backslash\{v\}$. (b) Consider the graph $\mathcal{F}=(V, E)$ and $S_{n}$ 's such that

$$
\begin{aligned}
V & =\left\{v_{(k, l)}: k, l \in \mathbb{Z}_{+}\right\}, \\
E & =\left\{\left(v_{(k, l)}, v_{(k+1, l)}\right),\left(v_{(k, l)}, v_{(k, l+1)}\right): k, l \in \mathbb{Z}_{+}\right\}, \\
S_{n} & =\left\{v_{(k, l+1)}: 0 \leq k \leq n-1,0 \leq l \leq n-1,\right\}, \quad n \in \mathbb{N}
\end{aligned}
$$

(see Fig. 3b). It this case (3) holds. However, $\bigcup_{n=1}^{\infty} S_{n}=V \backslash\left\{v_{(k, 0)}: k \in \mathbb{Z}_{+}\right\}$, so (2) is not satisfied.

(c) Let $\mathcal{F}=(V, E)$ be such that

$$
\begin{aligned}
& V=\{v\} \cup\left\{v_{(k, l)}: k, l \in \mathbb{Z}_{+}\right\} \\
& E=\left\{\left(v, v_{(k, 0)}\right): k \in \mathbb{Z}_{+}\right\} \cup\left\{\left(v_{(k, l)}, v_{(k, l+1)}\right): k, l \in \mathbb{Z}_{+}\right\} .
\end{aligned}
$$

For $n \in \mathbb{N}$, we set

$$
S_{n}=\{v\} \cup\left\{v_{(k, 0)}: k \in \mathbb{Z}_{+}\right\} \cup\left\{v_{(k, l)}: 0 \leq k \leq n-1,1 \leq l \leq n-k\right\} .
$$

The graph $\mathcal{F}$ with countable $S_{n}$ 's, illustrated in Fig. 3c, satisfies (1)-(4).

Before we move on, we need to introduce one more piece of notation. For $W \subset V$, we set

$$
W^{c}=\operatorname{Chi}(\operatorname{Par}(W)) \backslash W .
$$

It is easy to see that $\left(W^{c}\right)^{\circ}=W^{c}=\left(W^{\circ}\right)^{c}, \operatorname{Par}(W)=\operatorname{Par}\left(W^{\circ}\right)$ and

$$
\text { for } v \in V, \quad v \in W^{c} \quad \text { if and only if } \quad v \notin W \text { and } \operatorname{Par}(v) \cap \operatorname{Par}(W) \neq \emptyset \text {. }
$$

Theorem 7.4 Let $S_{\lambda}$ be a weighted shift on a directed semi-tree $\mathcal{F}=(V, E)$ with weights $\lambda=\left\{\lambda_{(u, v)}:(u, v) \in E\right\}$. Suppose that: 
(a)

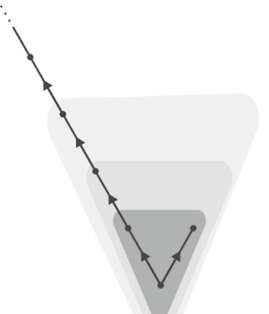

(b)

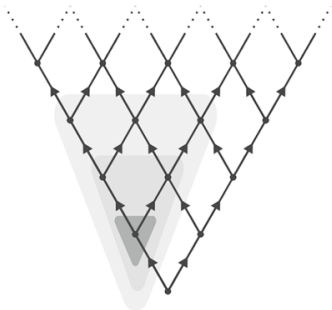

(c)

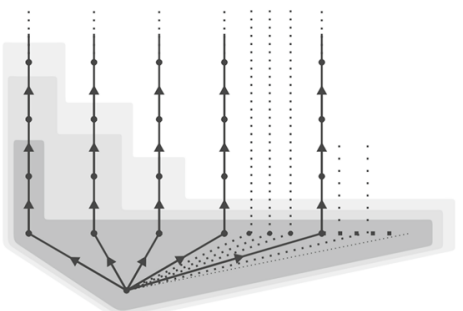

Fig. 3 Graphs illustrating Example 7.3

(i) $\mathcal{E}_{V} \subset \mathcal{D}\left(S_{\lambda}\right)$,

(ii) there exists a sequence $\left\{S_{n}\right\}_{n=1}^{\infty}$ satisfying (1)-(4) such that for each $f \in \mathcal{D}\left(S_{\lambda}\right)$, we have

$$
\lim _{n \rightarrow \infty} \sum_{v \in\left(S_{n}\right)^{c}}\left|\sum_{u \in \operatorname{Par}(v) \cap \operatorname{Par}\left(S_{n}\right)} \lambda_{(u, v)} f(u)\right|^{2}=0 .
$$

Then $S_{\lambda}=\overline{S_{\lambda} \mid \mathcal{E}_{V}}$.

Proof Take $f \in \mathcal{D}\left(S_{\lambda}\right)$ and set $f_{n}=\sum_{w \in \operatorname{Par}\left(S_{n}\right)} f(w) e_{w}$ for $n \in \mathbb{N}$. If $\operatorname{Par}\left(S_{n}\right)=\emptyset$, then $f_{n}=0$. Note that $f_{n} \in \mathcal{D}\left(S_{\lambda}\right)$, because each set $\operatorname{Par}\left(S_{n}\right)$ is finite and (i) holds. Since $S_{n} \subset S_{n+1}$, which yields $\operatorname{Par}\left(S_{n}\right) \subset \operatorname{Par}\left(S_{n+1}\right)$ for each $n \in \mathbb{N}$, and $V=$ $\bigcup_{n=1}^{\infty} \operatorname{Par}\left(S_{n}\right)$, it follows that $f_{n} \rightarrow f$ in $l^{2}(V)$ as $n \rightarrow \infty$. Next, observe that

$$
\begin{aligned}
\left(S_{\lambda} \mid \mathcal{E}_{V}\right) f_{n} & =\sum_{v \in V^{\circ}}\left[\sum_{u \in \operatorname{Par}(v)} \lambda_{(u, v)}\left(\sum_{w \in \operatorname{Par}\left(S_{n}\right)} f(w) e_{w}(u)\right)\right] e_{v} \\
& =\sum_{v \in V^{\circ}}\left[\sum_{u \in \operatorname{Par}(v) \cap \operatorname{Par}\left(S_{n}\right)} \lambda_{(u, v)} f(u)\right] e_{v} .
\end{aligned}
$$

Take $v \in V^{\circ}$. If $v \in S_{n}^{\circ}$, then $\operatorname{Par}(v) \subset \operatorname{Par}\left(S_{n}^{\circ}\right) \subset \operatorname{Par}\left(S_{n}\right)$. If $v \notin S_{n}^{\circ}$ and $\operatorname{Par}(v) \cap \operatorname{Par}\left(S_{n}\right) \neq \emptyset$, then equivalently $v \in\left(S_{n}\right)^{c}$ [by (7.1)]. From this and (7.3) we infer that

$$
\left(S_{\lambda} \mid \mathcal{E}_{V}\right) f_{n}=B_{1}\left(f_{n}\right)+B_{2}\left(f_{n}\right),
$$

where

$$
B_{1}\left(f_{n}\right):=\sum_{v \in S_{n}^{\circ}}\left[\sum_{u \in \operatorname{Par}(v)} \lambda_{(u, v)} f(u)\right] e_{v},
$$




$$
B_{2}\left(f_{n}\right):=\sum_{v \in\left(S_{n}\right)^{c}}\left[\sum_{u \in \operatorname{Par}(v) \cap \operatorname{Par}\left(S_{n}\right)} \lambda_{(u, v)} f(u)\right] e_{v} .
$$

Since $S_{n} \subset S_{n+1}$ for each $n \in \mathbb{N}$ and $V^{\circ}=\bigcup_{n=1}^{\infty} S_{n}^{\circ}$, it follows that $B_{1}\left(f_{n}\right) \rightarrow S_{\lambda} f$, while by the assumption (ii), $B_{2}\left(f_{n}\right) \rightarrow 0$ as $n \rightarrow \infty$. In view of (7.4), these facts imply that $\left(S_{\lambda} \mid \mathcal{E}_{V}\right) f_{n} \rightarrow S_{\lambda} f$ as $n \rightarrow \infty$, which completes the proof.

Remark 7.5 It should be noted that the above argument can serve as an alternative proof of Theorem 3.1.3 (iv) of [9] for a class of weighted shifts acting on directed trees of a special kind. Indeed, let $\mathcal{F}=(V, E)$ be a directed tree with a root. For $u \in V$, we set $l(u)=0$ if $u=$ root, $l(u)=1$ if (root, $u) \in E$, and $l(u)=n(n \geq 2)$ if there exists a finite sequence $u_{1}, \ldots, u_{n-1} \in V$ such that (root, $\left.u_{1}\right),\left(u_{1}, u_{2}\right), \ldots,\left(u_{n-1}, u\right) \in E$. Suppose additionally that $\mathcal{F}$ is such that $\operatorname{card}\left(S_{n}\right)<\aleph_{0}$ for each $n \in \mathbb{N}$, where

$$
S_{n}:=\{u \in V: l(u) \leq n\}
$$

Then $\operatorname{Par}\left(S_{n}\right)=S_{n-1}$ and $\operatorname{Chi}\left(S_{n}\right)=S_{n+1}$, so $\operatorname{Chi}\left(\operatorname{Par}\left(S_{n}\right)\right)=S_{n}(n \in \mathbb{N})$. It is also clear that $\left\{S_{n}\right\}_{n=1}^{\infty}$ satisfies conditions (1)-(4). Consider a densely defined weighted shift $S_{\lambda}$ on $\mathcal{F}$. In view of Theorem 3.1.3 (v) of [9], $\mathcal{E}_{V} \subset \mathcal{D}\left(S_{\lambda}\right)$. Next, note that condition (7.2) is trivially fulfilled, because $S_{n}^{c}=\emptyset(n \in \mathbb{N})$. As a result, we can apply Theorem 7.4 to deduce that $S_{\lambda}=\overline{S_{\lambda} \mid \mathcal{E}_{V}}$.

We close this paper by showing that each operator $\mathcal{A}_{a}^{+}$falls within the scope of Theorem 7.4.

Proposition 7.6 For each $a \in l^{2}, \mathcal{A}_{a}^{+}=\overline{\left.\mathcal{A}_{a}^{+}\right|_{\mathcal{E}_{V_{\infty}}}}$, where $\mathcal{E}_{V_{\infty}}$ is the linear span of the set $\left\{e_{\alpha}: \alpha \in V_{\infty}\right\}$.

Proof First observe that Proposition 6.3 implies the condition (i) of Theorem 7.4. We now show that the condition (ii) of Theorem 7.4 also holds. For $n \in \mathbb{N}$, we set

$$
S_{n}=\left\{\alpha \in V_{\infty}: \alpha=\left(\alpha_{1}, \ldots, \alpha_{n}, 0,0, \ldots\right) \text { and } \alpha_{1}+\cdots+\alpha_{n} \leq n+1\right\} .
$$

Then from Lemma 3.3 (iii) we deduce that

$$
\operatorname{Par}\left(S_{n}\right)=\left\{\beta \in V_{\infty}: \beta=\left(\beta_{1}, \ldots, \beta_{n}, 0,0, \ldots\right) \text { and } \beta_{1}+\cdots+\beta_{n} \leq n\right\}
$$
have

It is easily seen that the sequence $\left\{S_{n}\right\}_{n=1}^{\infty}$ fulfils conditions (1)-(4). Moreover, we

$$
\begin{aligned}
\left(S_{n}\right)^{c}= & \left\{\gamma \in V_{\infty}: \gamma=(\gamma_{1}, \ldots, \gamma_{n}, 0,0, \underbrace{1}_{j(\gamma)}, 0 \ldots), \text { where } j(\gamma)>n,\right. \\
& \text { and } \left.\gamma_{1}+\cdots+\gamma_{n} \leq n\right\} .
\end{aligned}
$$


Recall that, in view of the proof of Theorem 6.2, weights of the weighted shift $\mathcal{A}_{a}^{+}$are given by the formula $\lambda_{(\beta, \gamma)}=\overline{a_{i(\beta, \gamma)}} \sqrt{\gamma_{i(\beta, \gamma)}}$ for $(\beta, \gamma) \in E_{\infty}$, where $i(\beta, \gamma)$ is a unique natural number such that $\beta+\delta_{i(\beta, \gamma)}=\gamma$. Note also that if $\gamma \in\left(S_{n}\right)^{c}$, then $\operatorname{Par}(\gamma) \cap \operatorname{Par}\left(S_{n}\right)$ contains only one element such that $\gamma_{i(\beta, \gamma)}=1$ $(i(\beta, \gamma)=j(\gamma)>n)$. Consider the mapping

$$
p_{n}: V_{\infty} \ni\left(\alpha_{1}, \alpha_{2}, \ldots, \alpha_{n}, \alpha_{n+1}, \ldots\right) \mapsto\left(\alpha_{1}, \alpha_{2}, \ldots, \alpha_{n}, 0,0, \ldots\right) \in V_{\infty}
$$

Clearly, $p_{n}\left(S_{n}^{c}\right)=\operatorname{Par}\left(S_{n}\right)$. Taking all these into account, for $(f) \in \mathcal{D}\left(\mathcal{A}_{a}^{+}\right)$, we get

$$
\begin{aligned}
\sum_{\gamma \in\left(S_{n}\right)^{c}}\left|\sum_{\beta \in \operatorname{Par}(\gamma) \cap \operatorname{Par}\left(S_{n}\right)} \lambda_{(\beta, \gamma)} f_{\beta}\right|^{2} & =\sum_{\gamma \in\left(S_{n}\right)^{c}}\left|\overline{a_{i\left(p_{n}(\gamma), \gamma\right)}} f_{p_{n}(\gamma)}\right|^{2} \\
& =\sum_{\beta \in \operatorname{Par}\left(S_{n}\right)} \sum_{i=n+1}^{\infty}\left|\overline{a_{i}} f_{\beta}\right|^{2} \\
& =\left(\sum_{\beta \in \operatorname{Par}\left(S_{n}\right)}\left|f_{\beta}\right|^{2}\right)\left(\sum_{i=n+1}^{\infty}\left|a_{i}\right|^{2}\right) .
\end{aligned}
$$

Since

$$
\sum_{\beta \in \operatorname{Par}\left(S_{n}\right)}\left|f_{\beta}\right|^{2} \leq\|f\|^{2}<\infty
$$

and $a \in l^{2}$, from (7.5) we infer (7.2). Now we can appeal to Theorem 7.4 to finish the proof.

Acknowledgments The authors were supported by the Polish Ministry of Science and Higher Education (AGH local grant).

Open Access This article is distributed under the terms of the Creative Commons Attribution 4.0 International License (http://creativecommons.org/licenses/by/4.0/), which permits unrestricted use, distribution, and reproduction in any medium, provided you give appropriate credit to the original author(s) and the source, provide a link to the Creative Commons license, and indicate if changes were made.

\section{References}

1. Aronszajn, N.: Theory of reproducing kernels. Trans. Am. Math. Soc. 68, 337-404 (1950)

2. Bargmann, V.: On a Hilbert space of analytic functions and associated integral transform. I. Commun. Pure Appl. Math. 14, 187-214 (1961)

3. Bargmann, V.: Remarks on a Hilbert space of analytic functions. Proc. Natl. Acad. Sci. USA 48, 199-204 (1962)

4. Budzyński, P., Dymek, P., Jabłoński, Z.J., Stochel, J.: Subnormal weighted shifts on directed trees and composition operators in $L^{2}$-spaces with nondensely defined powers. Abstr. Appl. Anal. Art. ID. 791817 (2014)

5. Budzyński, P., Jabłoński, Z.J., Jung, I.B., Stochel, J.: Unbounded subnormal weighted shifts on directed trees. J. Math. Anal. Appl. 394, 819-834 (2012) 
6. Budzyński, P., Jabłoński, Z.J., Jung, I.B., Stochel, J.: Unbounded subnormal weighted shifts on directed trees. II. J. Math. Anal. Appl. 398, 600-608 (2013)

7. Fujii, J., Fujii, M., Sasaoka, H., Watatani, Y.: The spectrum of an infinite directed graph. Math. Jpn. 36, 607-625 (1991)

8. Fujii, J., Sasaoka, H., Watatani, Y.: Adjacency operators of infinite directed graphs. Math. Jpn. 34, 727-735 (1989)

9. Jabłoński, Z.J., Jung, I.B., Stochel, J.: Weighted shifts on directed trees. Mem. Am. Math. Soc. 1017, 107 (2012)

10. Jabłoński, Z.J., Jung, I.B., Stochel, J.: A non-hyponormal operator generating Stieltjes moment sequences. J. Funct. Anal. 262, 3946-3980 (2012)

11. Jabłoński, Z.J., Jung, I.B., Stochel, J.: Normal extensions escape from the class of weighted shifts on directed trees. Complex Anal. Oper. Theory 7, 409-419 (2013)

12. Jabłoński, Z.J., Jung, I.B., Stochel, J.: A hyponormal weighted shift on a directed tree whose square has trivial domain. Proc. Am. Math. Soc. 142, 3109-3116 (2014)

13. Mlak, W.: The Schrödinger type couples related to weighted shifts. Univ. Iagel. Acta Math. 27, 297-301 (1988)

14. Segal, I.E.: The complex wave representation of the free boson field. Adv. Math. Suppl. Stud. 3, 321-343 (1978)

15. Shields, A.L.: Weighted shift operators and analytic function theory. In: Topics in Operator Theory, pp. 49-128. Mathematical Surveys, No. 13, American Mathematical Society, Providence (1974)

16. Stochel, J.B.: A remark on Bargmann's Hilbert space of an infinite order. Opuscula Math. 10, 171-181 (1991)

17. Stochel, J.B.: Subnormality of generalized creation operators on Bargmann's space of infinite order. Integral Equ. Oper. Theory 15, 1011-1032 (1992)

18. Stochel, J.B.: A remark on Bargmann's space of an infinite order. II. Opuscula Math. 16, 97-110 (1996)

19. Stochel, J.B.: Representation of generalised creation and annihilation operators in Fock space. Univ. Iagel. Acta Math. 34, 135-148 (1997)

20. Stochel, J.B.: A note on inductive limit model of Bargmann space of infinite order. Opuscula Math. 25, 139-148 (2005)

21. Stochel, J.B.: A remark on generalized commutation relation and subnormality. Opuscula Math. 26, 151-160 (2006)

22. Stochel, J.B.: Erratum to: subnormality of generalized creation operators on Bargmann's space of infinite order. Integral Equ. Oper. Theory 73, 603-604 (2012) 